\title{
Foreword
}

Neuropsychobiology

\section{What Can Be Learned from Dimensional Perspectives on Psychiatry?}

\author{
Sebastian Walther ${ }^{a} \quad$ Manuel Morrens $s^{b, c}$ \\ ${ }^{a}$ Translational Research Center, University Hospital of Psychiatry, University of Bern, Bern, Switzerland; \\ ${ }^{b}$ Collaborative Antwerp Psychiatric Research Institute (CAPRI), Faculty of Medicine and Health Sciences, \\ Campus Drie Eiken, University of Antwerp, Antwerp, Belgium; ' ${ }^{2}$ niversity Department of Psychiatry, Campus Duffel, \\ Duffel, Belgium
}

When the field of psychiatry matured into a medical science, it was important to establish operationalized criteria and categories of disorders. This step enabled diagnostic comparisons across sites and countries and thus fostered studies on treatment evaluation in psychiatry [1]. The categories enjoyed high reliability. However, the diagnostic categories have fallen short in explaining variance in treatment outcomes, neuroimaging findings, blood-based biomarkers, or behavioral markers [2, 3]. Psychopathology spans in dimensions across different disorders, but also varies within a group of disorders as well as between subjects at risk and between subjects presenting a chronic condition. Finally, cognitive functions, affective states, and behaviors overlap between healthy subjects and patients with psychiatric disorders [4]. To make progress in psychiatry, it is critical to study psychopathological processes, as well as the underlying biology in a dimensional perspective spanning multiple disorders, stages, and normal experiences.

This special issue of Neuropsychobiology has addressed research on dimensional approaches to psychiatric illnesses. We have summarized ten contributions exploring dimensional perspectives in psychiatry.

The dimension of effortful control or self-regulation behavior is reviewed by Santens et al. [5]. This psycho- logical dimension spans normality as well as several distinct psychiatric diagnoses, such as mood disorders, anxiety, and substance use disorders. Similarly, Besteher et al. [6] summarized the evidence for grey matter alterations in subjects with subclinical symptoms of mood and anxiety disorders. Likewise, Geiser et al. [7] reviewed insomnia across diagnoses providing an in-depth discussion of the potential application of noninvasive brain stimulation techniques.

The motor dimension was explored in two studies. Ristanovic et al. [8] tested the association between motor abnormalities and cognition in subjects with nonclinical psychosis. Likewise, Kent et al. [9] investigated the association of motor behavior with psychopathological dimensions in a sample of 149 subjects including schizophrenia, schizoaffective disorder, bipolar disorder, relatives, and healthy subjects.

Hebbrecht et al. [10] tested symptom profiles in 276 inpatients with affective disorders to explore differences between major depression and bipolar depression. In contrast, Coppens et al. [11] studied a large number of candidate biomarkers that may help to discriminate unipolar from bipolar depression and schizophrenia.

Investigating subjects who are endorsing one prominent symptom also constitutes an interesting way of ex-

\section{KARGER}

(c) 2020 S. Karger AG, Basel 
ploring the neural correlates of symptoms in highly complex disorders such as schizophrenia. Wolf et al. [12] tested grey matter indices in subjects with persistent persecutory delusions. Likewise, Kubera et al. [13] examined resting state networks in subjects with persistent auditory verbal hallucinations. While Foucher et al. [14] integrated multiple levels of clinical and neuroimaging data to find a marker for a specific type of catatonia.

Finally, we discussed how immune dysregulations might affect psychotic and mood disorders [15]. In sum, this special issue represents very intriguing evidence supporting the dimensional perspective in psychiatric research. Much can be learned from studying behavior, bi- ology, and psychopathology considering a continuum from normality to severe disorders. This way of conducting psychiatric research is still in its infancy. Many useful findings are to be expected. This line of research is likely to stimulate research on novel treatment targets, which can be tested across different diagnoses, rendering large clinical trials possible. Such biomarkers can also be informative towards new diagnostic approaches. Eventually, dimensional perspectives on symptoms or behaviors will also strengthen efforts to fight stigma associated with psychiatric disorders, because it helps explain behaviors to the general population.

\section{References}

1 Carpenter WT Jr, Strauss JS, Bartko JJ. Flexible system for the diagnosis of schizophrenia: report from the WHO International Pilot Study of Schizophrenia. Science. 1973 Dec; 182(4118):1275-8.

2 Insel T, Cuthbert B, Garvey M, Heinssen R, Pine DS, Quinn K, et al. Research domain criteria (RDoC): toward a new classification framework for research on mental disorders. Am J Psychiatry. 2010 Jul;167(7):74851.

3 Strik W, Stegmayer K, Walther S, Dierks T. Systems Neuroscience of Psychosis: Mapping Schizophrenia Symptoms onto Brain Systems. Neuropsychobiology. 2017;75(3):10016.

4 Bebbington PE, McBride O, Steel C, Kuipers E, Radovanovic M, Brugha T, et al. The structure of paranoia in the general population. $\mathrm{Br}$ J Psychiatry. 2013 Jun;202(6):419-27.

5 Santens E, Claes L, Dierckx E, Dom G. Effortful Control - A Transdiagnostic Dimension Underlying Internalizing and Externalizing Psychopathology. Neuropsychobiology. 2020; 79:255-69.
6 Besteher B, Gaser C, Nenadić I. Brain Structure and Subclinical Symptoms: A Dimensional Perspective of Psychopathology in the Depression and Anxiety Spectrum. Neuropsychobiology. 2020;79:270-83.

7 Geiser T, Hertenstein E, Fehér K, Maier JG, Schneider CL, Züst MA, et al. Targeting arousal and sleep through non-invasive brain stimulation to improve mental health. Neuropsychobiology. 2020;79:284-92.

8 Ristanovic I, Osborne KJ, Vargas T, Gupta T, Mittal VA. Postural Control and Verbal and Visual Working Memory Correlates in Nonclinical Psychosis. Neuropsychobiology. 2020;79:293-300.

9 Kent JS, Disner SG, Van Voorhis AC, Urošević S, Caligiuri MP, Sponheim SR. Exploring the Relationship of Transdiagnostic Mood and Psychosis Symptom Domains with Motor Dysfunction. Neuropsychobiology. 2020;79:301-12.

10 Hebbrecht K, Stuivenga M, Birkenhäger T, van der Mast RC, Sabbe B, Giltay EJ. Symptom Profile and Clinical Course of Inpatients with Unipolar versus Bipolar Depression. Neuropsychobiology. 2020;79:313-23.
11 Coppens V, De Wachter O, Goossens J, Hendrix J, Maudsley S, Azmi A, et al. Profiling of the peripheral blood mononuclear cell proteome in schizophrenia and mood disorders for discovery of discriminatory biomarkers A proof of concept study. Neuropsychobiology. 2020;79:324-34.

12 Wolf RC, Hildebrandt V, Schmitgen MM, Pycha R, Kirchler E, Macina C, et al. Aberrant Gray Matter Volume and Cortical Surface in Paranoid-Type Delusional Disorder. Neuropsychobiology. 2020;79:335-44.

13 Kubera KM, Wolf ND, Rashidi M, Hirjak D, Northoff G, Schmitgen MM, et al. Functional decoupling of language and self-reference networks in patients with persistent auditory verbal hallucinations. Neuropsychobiology. 2020;79:345-51.

14 Foucher JR, de Billy C, Jeanjean LC, Obrecht A, Mainberger O, Clauss JM, et al. A Brain Imaging-Based Diagnostic Biomarker for Periodic Catatonia: Preliminary Evidence Using a Bayesian Approach. Neuropsychobiology. 2020;79:352-65.

15 Morrens M, Coppens V, Walther S. Do Immune Dysregulations and Oxidative Damage Drive Mood and Psychotic Disorders? Neuropsychobiology. 2020;79:251-54. 